\title{
Human Periodontal Ligament Derived Progenitor Cells: Effect of STRO-1 Cell Sorting and Wnt3a Treatment on Cell Behavior
}

\author{
Xiang-Zhen Yan, ${ }^{1,2}$ Sanne K. Both, ${ }^{1}$ Pi-Shan Yang, ${ }^{2}$ John A. Jansen,, \\ Jeroen J. J. P. van den Beucken, ${ }^{1}$ and Fang Yang ${ }^{1}$ \\ ${ }^{1}$ Department of Periodontology and Biomaterials, Radboud University Medical Center, 309 Dentistry, \\ P.O. Box 9101, 6500 HB Nijmegen, The Netherlands \\ ${ }^{2}$ Department of Periodontology, Shandong University, Jinan, Shandong 250012, China
}

Correspondence should be addressed to Fang Yang; fang.yang@radboudumc.nl

Received 11 February 2014; Revised 8 April 2014; Accepted 12 April 2014; Published 28 April 2014

Academic Editor: Jun Liao

Copyright (c) 2014 Xiang-Zhen Yan et al. This is an open access article distributed under the Creative Commons Attribution License, which permits unrestricted use, distribution, and reproduction in any medium, provided the original work is properly cited.

\begin{abstract}
Objectives. STRO-1 positive periodontal ligament cells (PDLCs) and unsorted PDLCs have demonstrated potential for periodontal regeneration, but the comparison between unsorted cells and the expanded STRO-1 sorted cells has never been reported. Additionally, Wnt3a is involved in cell proliferation thus may benefit in vitro PDLC expansion. The aim was to evaluate the effect of STRO-1 cell sorting and Wnt3a treatment on cell behavior of human PDLCs (hPDLCs). Materials and Methods. STRO-1 positive hPDLCs were sorted and the sorted cells were expanded and compared with their unsorted parental cells. Thereafter, hPDLCs were treated with or without Wnt3a and the cell proliferation, self-renewal, and osteogenic differentiation were evaluated. Results. No differences were measured between the expanded STRO-1-sorted cells and unsorted parental cells in terms of proliferation, $\mathrm{CFU}$, and mineralization capacity. Wnt3a enhanced the proliferation and self-renewal ability of hPDLCs significantly as displayed by higher DNA content values, a shorter cell population doubling time, and higher expression of the self-renewal gene Oct4. Moreover, Wnt3a promoted the expansion of hPDLCs for 5 passages without affecting cell proliferation, CFU, and osteogenic capacity. Conclusions. Expanded STRO-1-sorted hPDLCs showed no superiority compared to their unsorted parental cells. On the other hand, Wnt3a promotes the efficient hPDLC expansion and retains the self-renewal and osteogenic differentiation capacity.
\end{abstract}

\section{Introduction}

Periodontitis is a multifactorial disease caused primarily by dental plaque microorganisms [1]. Periodontitis is characterized by the destruction of the periodontium, including gingiva, periodontal ligament (PDL), cementum, and alveolar bone. Without adequate treatment, periodontitis will finally lead to tooth loss, which often affects nutrition intake and self-confidence. Approximately $48 \%$ of adults have chronic periodontitis and advanced periodontitis is more prevalent among the older age groups $[1,2]$. Current treatments are generally successful in preventing active disease, but the regeneration of the lost tissues remains a challenge. Recently, substantial progress has been made in periodontal tissue regeneration by cytotherapeutic approaches to overcome the limitations of existing procedures [3-5].
Several cell types have been used for periodontal regeneration including periodontal ligament cells (PDLCs), bone marrow stromal cells (BMSCs), alveolar periosteal cells (APCs), dental follicle cells (DFCs), and dental pulp cells (DPCs) [3, 5-7]. Tsumanuma et al. transplanted PDLCs, BMSCs, and APCs in canine one-wall intrabony defects for eight weeks [6] and results showed that significantly more newly formed cementum and well-oriented PDL fibers were formed in the PDLCs group than in the other groups. Besides, in an organ culture study performed on tooth root surfaces, new alveolar bone and PDL-like tissues were formed only by PDLCs but not by DFCs, DPCs, or BMSCs [7]. These results indicate that PDLCs may be the most suitable cell source for periodontal tissue regeneration.

STRO-1, one of the most well-known mesenchymal stemcell markers, has gained increasing interest in stem cell 
sorting over the past decade [7-11]. For instance, STRO-1 has been utilized for the selection of PDL stem cells [8], dental pulp stem cells $[7,9]$, and adipose-derived stem cells [10]. STRO-1 positive PDL stem cells are usually utilized for research purpose and their potential to regenerate periodontal tissues in vivo has been reported [8]. Since PDLCs contain subpopulations of stem cells [12], the heterogeneous unsorted PDLCs have also been shown to promote periodontal tissue formation $[5,7,9,10]$. The sorted stem cells in high purity might provide a better cell source for therapeutic purposes compared with the heterogeneous unsorted cells. But STRO1 positive cells are usually found in low numbers $[13,14]$ and therefore in vitro expansion is needed. However, the expression of STRO-1 was gradually lost during culture expansion, as suggested in previous studies $[13,14]$. Yet, the comparison between unsorted parental cells and the expanded STRO-1 sorted cells (equal expansion as the parental cells) has never been reported. Moreover, from a practical point of view, the cell selection and expansion procedure are time-consuming. Thus, it is of importance to compare unsorted parental cells and the expanded STRO-1 sorted cells from PDLCs in order to benefit their future clinical applications.

Along with the high quality, large quantity of cells is necessary for effective therapeutic applications. For instance, 160 million cells would be required for 20 cubic centimeter of tissue engineered bone implant based on using 8 million cells $/ \mathrm{cm}^{3}$ scaffold $[15,16]$ to gain substantial bone formation. PDLCs are easily accessible but the cell number is very limited from primary cell culture, and hence it requires in vitro expansion before clinical applications. Yet characteristic changes of PDLCs have been observed during passaging [13]. Alkaline phosphatase (ALP) activity of PDLCs gradually decreased as the passage number increased [13]. Thus, finding a method that can benefit the efficient in vitro expansion of PDLCs is required.

The cellular signaling pathways that control the proliferation of PDLCs are unclear. A promising candidate is the Wnt signaling, which is involved in tooth development $[17,18]$. Wnt signaling affects tooth size [17] and induces continuous tooth generation in mouse [18]. As reported in a previous study [19], in a continuously erupting tooth, cells with the highest level of Wnt responsiveness also show the highest proliferation. Wnt3a, a representative canonical Wnt member, has been recently isolated as an active Wnt molecule [20]. Wnt3a can enhance clonal outgrowth of neural stem cells [21] and promote long-term expansion of mammary stem cells [22]. Furthermore, Wnt signaling has been suggested to play an essential role in osteogenesis in vitro and in vivo [23-25]. For example, Wnt3a suppressed osteogenic differentiation of mesenchymal stem cells (MSCs) [23]. However, whether Wnt3a can directly control the proliferation and differentiation of adult human PDLCs is not known.

The aim of this study was to evaluate the effect of STRO-1 cell sorting and Wnt3a treatment on cell behavior of human PDLCs (hPDLCs). To this end, STRO-1 positive PDLCs were sorted and then the sorted cells were expanded and compared with their unsorted parental cells in terms of proliferation, colony forming unit (CFU), and mineralization. Thereafter, hPDLCs were treated with or without Wnt3a and the cell proliferation, self-renewal, and osteogenic differentiation were evaluated. It was hypothesized that Wnt3a can benefit the efficient in vitro expansion of hPDLCs by enhancing cell proliferation and self-renewal, while inhibiting osteogenic differentiation.

\section{Materials and Methods}

2.1. Cell Isolation. All experiments were done by following national guidelines for working with human materials (Dutch federation of biomedical scientific societies. Human tissue and medical research: code of conduct for responsible use, available at: http://www.federa.org/). After patients had signed informed consent, adult hPDLCs from 3 patients $(20,24$, and 33 years old, resp.) were obtained from healthy impacted third molars, which were routinely extracted for the prevention of third molar-related morbidity. The PDL was scraped from the middle third of the roots, dissected, and placed in a $25 \mathrm{~cm}^{2}$ culture flask in proliferation medium, which contained $\alpha$-MEM (Gibco) supplemented with $10 \%$ FCS (Gibco) and 100 units/mL pen/strep (Gibco). Bone was harvested from the posterior maxilla of 1 patient (41 years old) during dental implant surgery. Bone debris was retrieved from the drill surface and chopped into small pieces and put into a $50 \mathrm{~mL}$ tube. The tube with minced bone debris and proliferation medium was shaken vigorously and medium with human bone marrow stromal cells (hBMSCs) was collected and plated in a $25 \mathrm{~cm}^{2}$ culture flask. Upon $70-80 \%$ confluency, hPDLCs and hBMSCs were subcultured for 3 passages and afterwards cryopreserved in $\alpha$-MEM containing $20 \%$ FCS and 10\% DMSO (Sigma).

2.2. Cell Characterization. After defrosting, the morphology of hPDLCs was observed by an inverse phase contrast microscope (Leica DMIL, Germany) at $\times 10$ magnification. The self-renewal and osteogenic ability of hPDLCs from the 4th passage were characterized by colony-forming unit (CFU) efficiency, ALP activity, and mineralization ability (Von-Kossa staining). CFU assay was performed on day 10 in proliferation medium. ALP activity of hPDLCs was measured on day 8 in osteogenic medium (proliferation medium containing $50 \mu \mathrm{g} / \mathrm{mL}$ ascorbic acid, $10 \mathrm{mM}$ sodium $\beta$-glycerophosphate, and $10 \mathrm{nM}$ dexamethason, all from Sigma). Human fibroblasts from foreskin (a kind gift from Department of Orthodontics and Oral Biology, Radboud University Medical Centre) and hBMSCs were used as negative and positive control for ALP activity assay. Von-Kossa staining was performed after 30 days of culture in osteogenic medium. All the assays are described in detail below.

\subsection{STRO-1 Fluorescence-Activated Cell Sorting and Testing.} The cryopreserved hPDLCs from 2 donors were defrosted and performed for cell sorting experiments. Single hPDLC suspensions containing $10 \times 10^{6}$ cells from the 4 th passage were obtained through a $70 \mu \mathrm{m}$ cell strainer (Falcon BD, Franklin lakes, NJ, USA). Next, the cell suspensions were centrifuged at $400 \mathrm{~g}$ for $10 \mathrm{~min}$, resuspended in $1 \mathrm{~mL} \mathrm{PBS} / 1 \% \mathrm{BSA}$, 
and preincubated for $20 \mathrm{~min}$ on ice. Subsequently, cells were incubated with $200 \mu \mathrm{L}$ mouse anti-human monoclonal STRO-1 IgM primary antibody $\left(2.5 \mu \mathrm{g} / 10^{6}\right.$ cells, R\&D Systems, Minneapolis, MN) in PBS/1\%BSA for 15 min on ice and washed 3 times with PBS followed by centrifugation at $250 \mathrm{~g}$ for 5 minutes. The cell pellet was resuspended in $200 \mu \mathrm{L}$ $\mathrm{PBS} / 1 \% \mathrm{BSA}$ with $10 \mu \mathrm{L}$ phycoerythrin- (PE-) conjugated goat anti-mouse IgM antibody (R\&D Systems, Minneapolis, MN) for $30 \mathrm{~min}$ on ice, washed for 3 times, resolved in $400 \mu \mathrm{L}$ of PBS, and kept on ice until sorting. For blank control, PBS was substituted for the primary and second antibodies. To confirm the specificity of primary antibody binding, nonspecific mouse IgM isotype control (lambda monoclonal, abcam) which ideally matches the primary antibody's host species was substituted for the primary antibody. All incubations were performed in the dark at $4^{\circ} \mathrm{C}$. Cells were sorted using a FACStar Plus flow cytometer (Beckton Dickinson \& Co., Mountain View, CA). Positivity was defined as a level of fluorescence greater than $99 \%$ of the blank (without 1st and 2nd antibodies) and negative (without 1st antibody) control. To prove the efficacy of the applied FACS sorting, sorted cells and controls (STRO-1 ${ }^{+} /$STRO-1 $1^{-} /$un-sorted cells) were evaluated by fluorescence microscopy and flow cytometry. STRO $-1^{+}$cells were collected and expanded to $10 \times 10^{6}$ cells in proliferation medium. The expanded cells were compared with their unsorted parental cells in terms of proliferation (DNA content on days 2, 4, and 6 in proliferation medium), CFU ability (day 10 in proliferation medium), and mineralization capacity (calcium content on day 30 in osteogenic medium). All the assays are described in detail below.

2.4. Direct Effect of Wnt3a on hPDLCs. Based on the STRO1 cell sorting results, unsorted hPDLCs at 4 th passage were used in this study. hPDLCs from 3 donors were treated with or without $50 \mathrm{ng} / \mathrm{mL}$ Wnt3a (R\&D Systems). The dosage was chosen according to a previous study in which $50 \mathrm{ng} / \mathrm{mL}$ Wnt3a promoted long-term expansion of mammary stem cells [22]. Then, DNA content, gene expression (self-renewal gene markers Oct4, Nanog and Sox2, and osteogenic gene markers ALP, Runx-2, and OC), ALP activity, and calcium content were evaluated. All the assays are described in detail below.

2.5. Functionality of PDLCs Pretreated with Wnt3a. For a long-term study, hPDLCs from passage 4 were subcultured with or without $50 \mathrm{ng} / \mathrm{mL}$ Wnt3a up to passage 9 , and at each passage the cell doubling time was calculated. After pretreatment with or without Wnt3a for 5 passages, the functionality of Wnt3a-pretreated and control cells was compared in terms of proliferation (DNA content), CFU, and osteogenic differentiation (ALP activity and calcium content). All the assays are described in detail below.

2.6. DNA Assay. After 2, 4, and 6 days of incubation in proliferation medium, samples were prepared by washing the cells layers twice with PBS and adding $1 \mathrm{~mL}$ of MilliQ to each well, after which repetitive freezing $\left(-80^{\circ} \mathrm{C}\right)$ and thawing $\left(37^{\circ} \mathrm{C}\right)$ cycles were performed. DNA analysis was performed via a PicoGreen dsDNA quantification kit (Molecular
Probes, Leiden, The Netherlands) following manufacturer's instructions. Briefly, $100 \mu \mathrm{L}$ of DNA standard or sample was incubated with $100 \mu \mathrm{L}$ of working solution for $10 \mathrm{~min}$ at RT in the dark. After incubation, DNA content was measured using a fluorescence microplate reader (Bio-Tek Instruments, Abcoude, The Netherlands) with excitation filter $485 \mathrm{~nm}$ and emission filter $530 \mathrm{~nm}$.

2.7. ALP Activity. Cells in osteogenic medium were harvested at days 7 and 10 in the same way as cells for the DNA content. Then, $100 \mu \mathrm{L}$ of substrate solution (p-nitrophenyl phosphate) was added to $20 \mu \mathrm{L}$ of buffer (0.5 M 2-amino-2-methyl-1propanol) and $80 \mu \mathrm{L}$ of sample or standard in a 96-well plate. The standards were made by serial dilutions of 4-nitrophenol at final concentrations of $0-25 \mathrm{nM}$. The plate was incubated at $37^{\circ} \mathrm{C}$ for 1 hour. The reaction was terminated by adding $100 \mu \mathrm{L}$ of $0.3 \mathrm{M} \mathrm{NaOH}$. The absorbance of each well was measured in an ELISA microplate reader (Bio-Tek Instruments, Abcoude, The Netherlands) at $405 \mathrm{~nm}$. ALP activity was normalized to the amount of DNA.

2.8. Calcium Content. After 30 days of culture in osteogenic medium, cells were washed twice with PBS and then $1 \mathrm{~mL}$ of $0.5 \mathrm{~N}$ acetic acid was added to the each well. The tissue culture plate was incubated overnight on a shaking table. The calcium content was measured by the ortho-cresolphthalein complexone (OCPC) method (Sigma). For the biochemical assay, $10 \mu \mathrm{L}$ samples or standards were incubated with $300 \mu \mathrm{L}$ of working solution (Genzyme Diagnostics, Cambridge, MA, USA) in a 96-well plate. Standards $(0-100 \mu \mathrm{g} / \mathrm{mL})$ were generated using a $\mathrm{CaCl}_{2}$ stock solution. The plate was incubated at RT for $10 \mathrm{~min}$ and then the absorbance of each well was measured in the ELISA microplate reader (Bio-Tek Instruments, Abcoude, The Netherlands) at $570 \mathrm{~nm}$.

2.9. Von Kossa Staining. After 30 days of osteogenic induction, cells were fixed in $10 \%$ formalin for $20 \mathrm{~min}$, rinsed with MilliQ, and then stained with $5 \%$ silver nitrate $\left(\mathrm{AgNO}_{3}\right.$, Merck) for $30 \mathrm{~min}$. After washing with distilled water, the staining was developed with $5 \%$ sodium carbonate $\left(\mathrm{Na}_{2} \mathrm{CO}_{3}\right.$, Merck), fixed with $5 \%$ sodium thiosulphate $\left(\mathrm{Na}_{2} \mathrm{~S}_{2} \mathrm{O}_{3}\right.$, Merk), and examined by using a stereomicroscope (Leica MZ12, Germany).

2.10. CFU Assay. Single cell suspensions (1000 cells/mL; $100 \mu \mathrm{L}$ ) were seeded into one well of a 6-well plate in proliferation medium. After 10 days, the samples were fixated with $10 \%$ formalin and stained with $0.1 \%$ toluidine blue (Sigma, Chemical Co., St. Louis, MO, USA). Colony-forming efficiency (an aggregate of $\geq 50$ cells was scored as a colony) was determined by the number of colonies relative to the total number of seeded cells in each plate using a microscope (Leica DMIL, Germany).

2.11. Real-Time PCR. Cells in proliferation medium at day 5 (Oct4, Nanog, and Sox2 gene expression) and in osteogenic medium at days 7 and 14 (ALP, Runx-2, and $O C$ gene expression) were washed twice with PBS. Total RNA was 
extracted using TRIzol reagent (Invitrogen, Breda, The Netherlands) following manufacturer's instructions. RNA concentrations and purity were determined by NanoDrop (ND-2000, Thermo Scientific). Then, the reverse transcriptase (RT) reaction of $1 \mu \mathrm{g}$ RNA for each sample was performed using the Superscript III First-strand Synthesis System (Invitrogen, Breda, the Netherlands) for RT-PCR. The cDNA was amplified and gene expression was quantified with real-time PCR (BIORAD, CFX96 real-time system). The primers used were Oct4, Nanog, Sox2, ALP, Runx-2, and OC (sequences in Table 1). The expression levels were analyzed versus the housekeeping gene GAPDH. The specificity of the primers was tested before the real-time PCR reaction. IQ SYBR Green Supermix PCR kit (BioRad, Hemel Hempstead, United Kingdom) was used for real-time measurement. The melting temperature $(\mathrm{Tm})$ employed for each primer pair was $60^{\circ} \mathrm{C}$. The gene expression was calculated using the $2^{-\Delta \Delta \mathrm{Ct}}$ method and the control was used as the calibrator group.

2.12. Statistical Analysis. Each assay was performed in triplicate for each donor and statistical analysis was performed using GraphPad InStat (GraphPad Software, San Diego California USA). Results were statistically evaluated for each donor using an unpaired $t$-test (significance level, $P<0.05$ ).

\section{Results}

As all the donors showed the similar trend, only the result from one donor was presented below unless specifically mentioned.

3.1. Characterization of $h P D L C s$. hPDLCs were characterized on basis of their morphology, self-renewal, and osteogenic ability. Microscopy observation revealed spindle-shape cell morphology. hPDLCs displayed the ability to form colonies when cultured in proliferation medium after 10 days. ALP activity was confirmed in the hPDLC culture after 8 days in osteogenic medium. The ALP activity in hPDLCs was in between hBMSCs (positive control) and fibroblasts (negative control) cultured at the same conditions (Figure 1(a)). hPDLCs induced mineralization as determined by Von Kossa staining after 30 days of osteogenic induction (Figure 1(b)).

\subsection{Comparison between Expanded STRO-1-Sorted Cells and} Unsorted Parental Cells. The efficiency of FACS sorting was proved by fluorescent microscopy observation and flow cytometry. The STRO $-1^{+}$cells were expanded for 4 passages to achieve $10 \times 10^{6}$ cells, the same number as their unsorted parental cells. During this expansion, the percentage of STRO- $1^{+}$cells decreased significantly from $95.3 \%$ to $2.3 \%$ (Figure 2(a)). Additionally, after 4 passages of in vitro expansion, no significant difference was observed between the expanded STRO-1-sorted cells and unsorted parental cells in terms of DNA content, CFU number, and calcium content (Figures 2(b)-2(d)).

3.3. The Direct Effect of Wnt3a on hPDLCs. The effect of Wnt3a on hPDLC proliferation was assessed by measuring

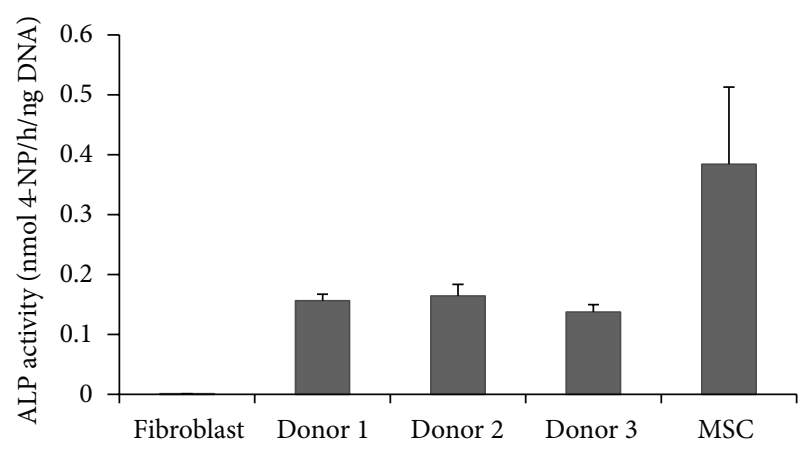

(a)

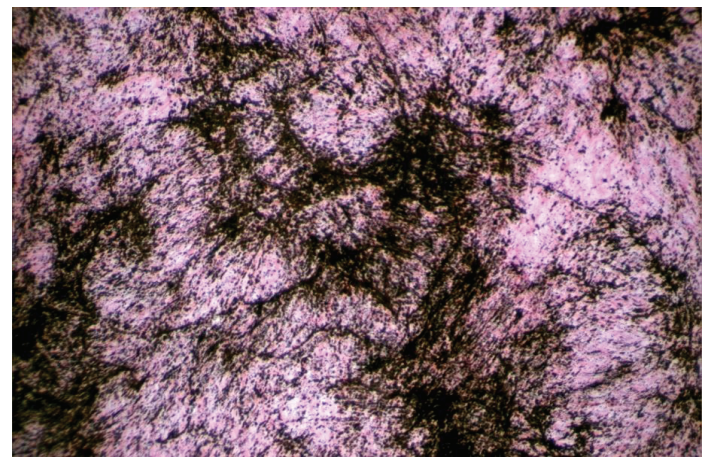

(b)

FIGURE 1: Characterization of hPDLCs. (a) ALP activity of the hPDLCs was lower compared to hBMSCs (positive control) on day 8, while fibroblasts were negative. (b) Mineral deposition was detected by a Von Kossa staining (black color) after 30 days of osteogenic induction. Error bars represent standard deviation.

cell DNA content and calculating cell doubling time during passaging. Wnt 3 a treated cells exhibited a significant increase $(P<0.05$; around $50 \%$ increase for day 4 and day 6 in DNA content compared to the nontreated control group; Figure 3(a)). In addition, Wnt3a-treated cells displayed significantly shorter population doubling time when compared to untreated control cells, which were $25.46 \pm 3.76$ versus $30.73 \pm 0.9$ hours $(P<0.05)$.

The self-renewal ability was evaluated by the expression of the self-renewal genes octamer-binding transcription factor 4 (Oct4), Nanog, and sex determining region Y-box 2 (Sox2). The mRNA expression of Oct4 was significantly higher $(P<$ 0.05 ) for the cells treated with Wnt3a than for the control cells (Figure 3(b)). Wnt3a-treated cells also exhibited higher Nanog and Sox2 expression, but a significant difference was only observed in one donor.

Osteogenic differentiation was assessed by gene expression of $A L P$, runt-related transcription factor 2 (Runx-2), and osteocalcin $(O C)$, ALP activity, calcium content in the extracellular matrix, and Von Kossa staining. PCR revealed significantly lower $(P<0.05) A L P$ gene expression for Wnt3a-treated cells at day 7 compared to the control group (Figure 4(a)). For the Runx-2 and OC gene expression no significant difference was found at day 7 and day 14 between the Wnt3a-treated and control groups (Figures 4(a) and 4(b)). 
TABle 1: Primer sequences used for real-time qPCR.

\begin{tabular}{lcc}
\hline & Forward $\left(5^{\prime} \rightarrow 3^{\prime}\right)$ & Reverse $\left(5^{\prime} \rightarrow 3^{\prime}\right)$ \\
\hline Oct4 & TTGCCCTTCTGGCGCCGGTTA & GTCAGGCCCGTCTCAGCTCATTG \\
Nanog & TTGTCCCCAAAGCTTGCCTTGCT 2 & TTCTTACCAGTCTCCGTGTGAGGC \\
ALP & AAAAACAGCCCGGACCGCGT & TCGTCGATGAACGGCCGCTT \\
Run $x-2$ & GGGACTGGTACTCGGATAACGA & CTGATATGCGATGTCCTTGCA \\
OC & GAGCACAAACATGGCTGAGA & TGGAGATGTTGCTCTGTTCG \\
GAPDH & AGGGCAGCGAGGTAGTGAAGA & TAGACCGGGCCGTAGAAGC \\
& CGATGCTGGCGCTGAGTAC & CGTTCAGCTCAGGGATGACC \\
\hline
\end{tabular}

[A] PMT3 log/FS Lin

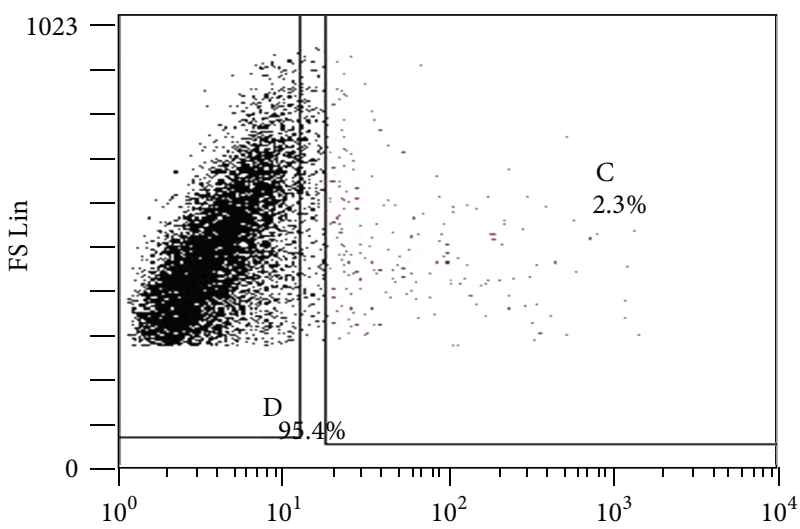

(a)

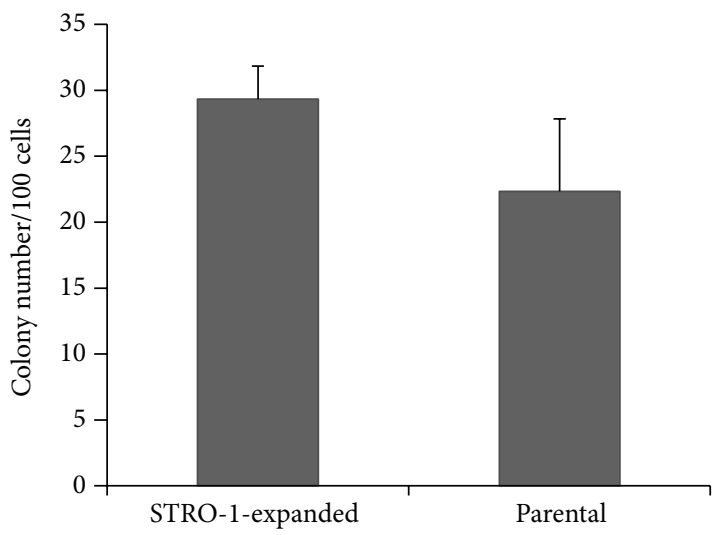

(c)

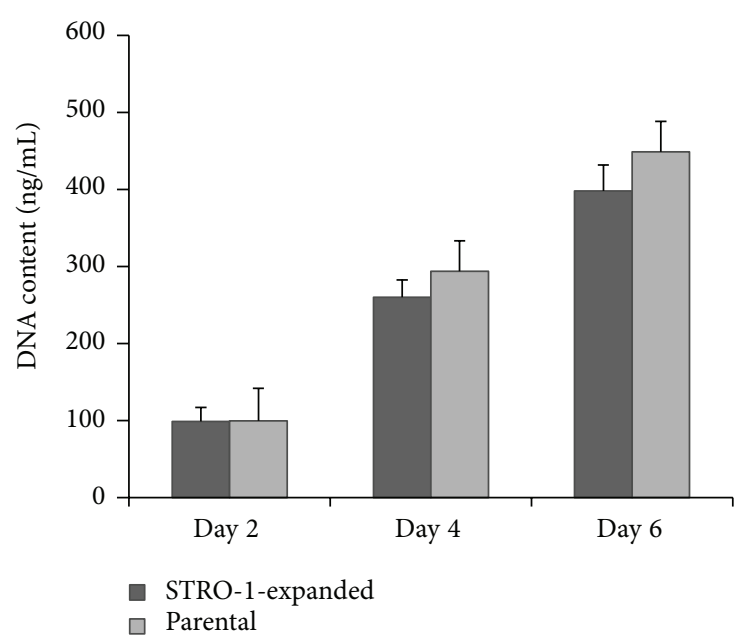

(b)

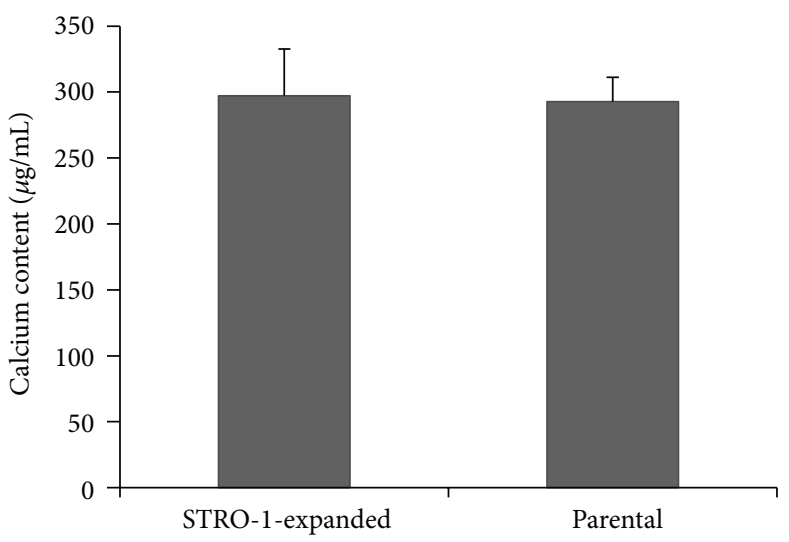

(d)

FIGURE 2: Representative results of STRO-1 cell sorting. (a) The percentage of sorted STRO- $1^{+}$cells decreased to $2.3 \%$ after 4 passages of in vitro expansion. (b) No difference in proliferation was observed between the expanded STRO-1-sorted cells and the unsorted parental cells. (c) Expanded STRO-1-sorted cells displayed no difference compared to the unsorted parental cells in colony forming number. (d) No difference in calcium content was observed between expanded STRO-1-sorted cells and parental hPDLCs. Error bars represent standard deviation.

ALP activity at days 7 and 10 demonstrated significantly lower $(P<0.05)$ levels for Wnt3a-treated cells versus untreated cells (Figure $4(c)$ ). The long-term mineralization was evaluated by calcium content deposited by the cells and a Von Kossa staining after 30 days of culture. Both Wnt3a treated and untreated cells induced mineralization as determined by
Von Kossa staining and no significant difference in calcium content was observed between the two groups (Figure 4(d)).

3.4. Functionality of Wnt3a Pretreated hPDLCs. After passage 9, Wnt3a was no longer added to the culture medium and the proliferation, CFU, and osteogenic differentiation capacity 


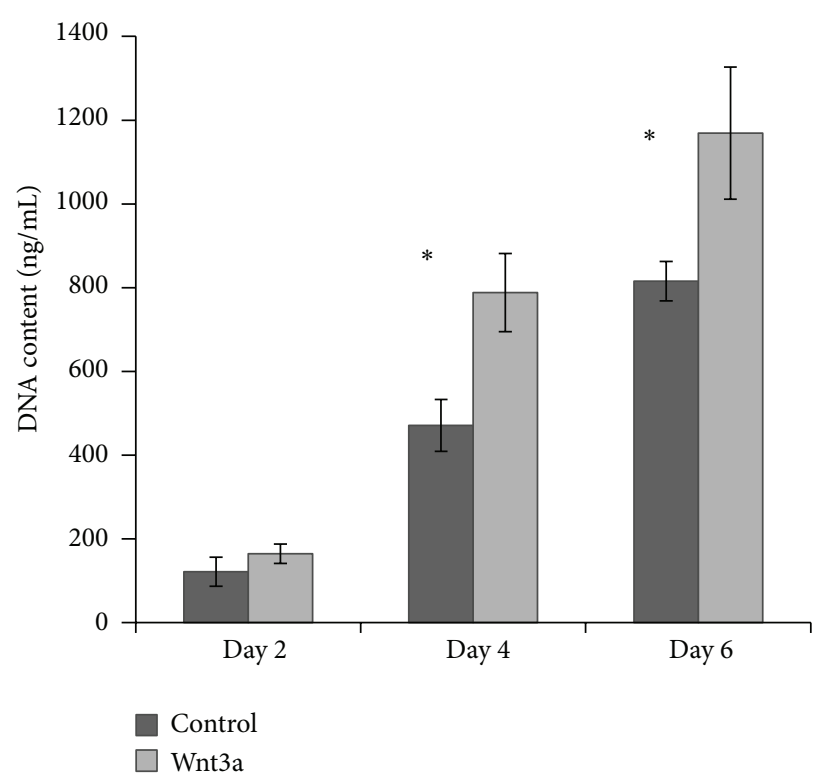

(a)

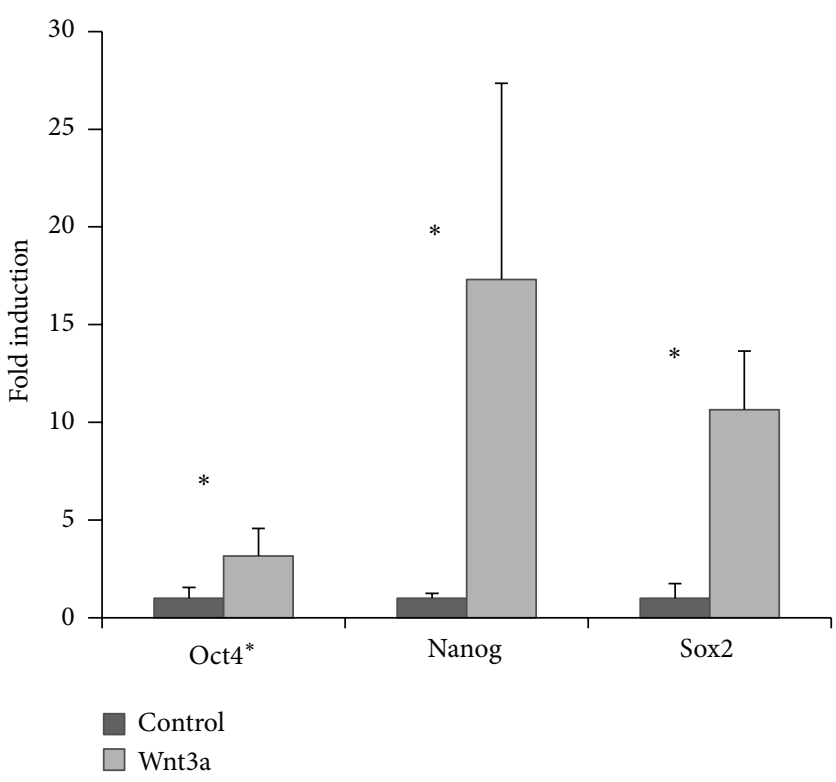

(b)

FIGURE 3: Representative results showed Wnt3a promoted proliferation and self-renewal ability of hPDLCs. (a) Wnt3a-treated cells displayed a significant increase in DNA content compared to untreated cells cultured in proliferation medium. (b) Wnt3a-treated cells exhibited a higher expression of Oct4, Nanog, and Sox 2 after 5 days of culture. ${ }^{*} P<0.05$; error bars represent standard deviation.

were investigated to evaluate whether the functionality of Wnt3a-pretreated cells was affected by the shorter cell doubling time. The results showed that both Wnt3a-pretreated cells and control cells showed abilities to proliferate, form colonies, and osteogenically differentiate. In addition, no difference was observed between Wnt3a-pretreated cells and control cells in terms of DNA content (Figure 5(a)), CFU number (Figure 5(b)), ALP activity (Figure 5(c)), or calcium content (Figure 5(d)).

\section{Discussion}

The aim of the current study was to evaluate the effect of STRO-1 cell sorting and Wnt3a treatment on cell behavior of hPDLCs. The characteristics of unsorted parental cells and expanded STRO-1-sorted cells were compared to find the appropriate PDL cell population for clinical applications. The effect of Wnt3a on proliferation, self-renewal, and osteogenesis of hPDLCs was evaluated to test the potential of Wnt3a for in vitro expansion of hPDLCs. No differences were measured between the expanded STRO-1-sorted cells and unsorted parental cells in terms of proliferation, CFU, and mineralization capacity. Wnt3a promoted the proliferation and self-renewal ability of hPDLCs, as displayed by an increased DNA content, shorter population doubling time, and higher expression of Oct4. Wnt3a also stimulated the efficient in vitro expansion of hPDLCs for at least 5 passages without affecting cell functionalities in terms of proliferation, $\mathrm{CFU}$, and osteogenic capacity.

STRO-1 positive cells are usually found in low numbers [13] and therefore in vitro expansion is needed. Our results showed that the percentage of STRO-1 positive cells decreased significantly during culture expansion. Additionally, after 4 passages of in vitro expansion, there were no differences in proliferation, CFU ability, and mineralization capacity between sorted and initially unsorted cells. A similar phenomenon has been observed with hBMSCs (unpublished data): the STRO-1 sorted hBMSCs returned to the same state as their unsorted parental cells only in 7 days of in vitro expansion. Based on the results and also the time-consuming procedure of the cell selection and expansion, unsorted primary cells may provide a better cell source for future clinical applications. Therefore, unsorted hPDLCs were used in the current study to test the effect of Wnt3a.

The outcome of DNA content indicates that Wnt3a enhances the proliferation of hPDLCs significantly. Additionally during the long-term study, the cells were treated with or without Wnt3a for 5 passages with the same cell seeding density. Wnt3a-treated cells showed more cell doublings than the control group at each passage, indicating the enhancement of Wnt3a on the proliferation of hPDLCs. Oct4, Nanog, and Sox2 are key regulators essential for self-renewal of pluripotent cells [26]. These self-renewal factors are also expressed in hPDLCs $[12,27]$ and thus they were chosen in this study. Based upon the results, the self-renewal ability of hPDLCs was promoted by Wnt3a as displayed by the higher expression of self-renewal gene expressions. This is consistent with one previous study in which self-renewal and gene expression were stimulated in embryonic stem cells by adding Wnt3a [28]. These results do suggest that Wnt3a may benefit the in vitro expansion of primary cells for further cell-based clinical applications.

Our results further suggest that Wnt3a mediates some aspects of osteogenic differentiation. ALP mRNA-levels and 


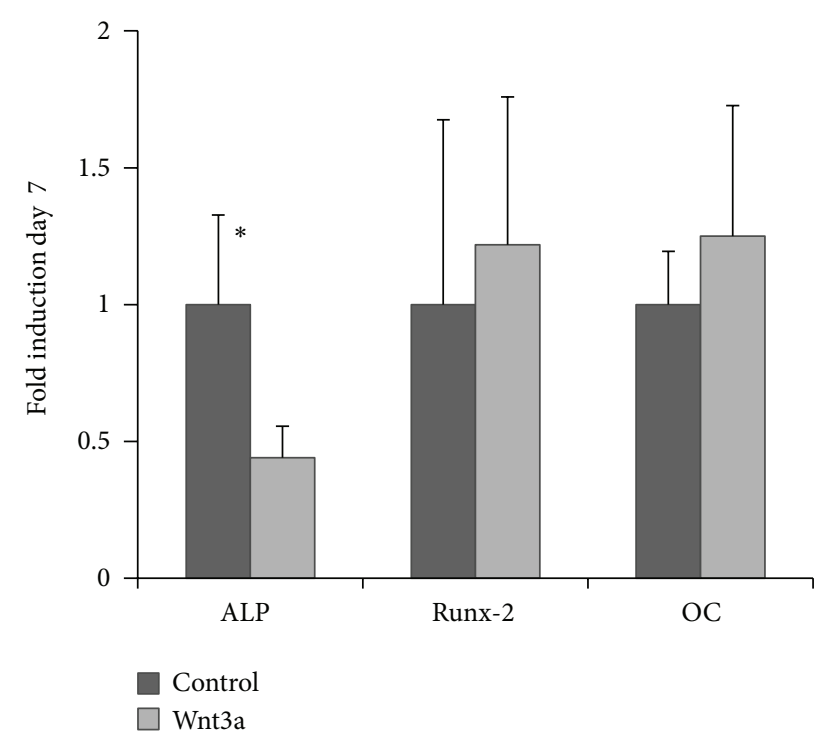

(a)

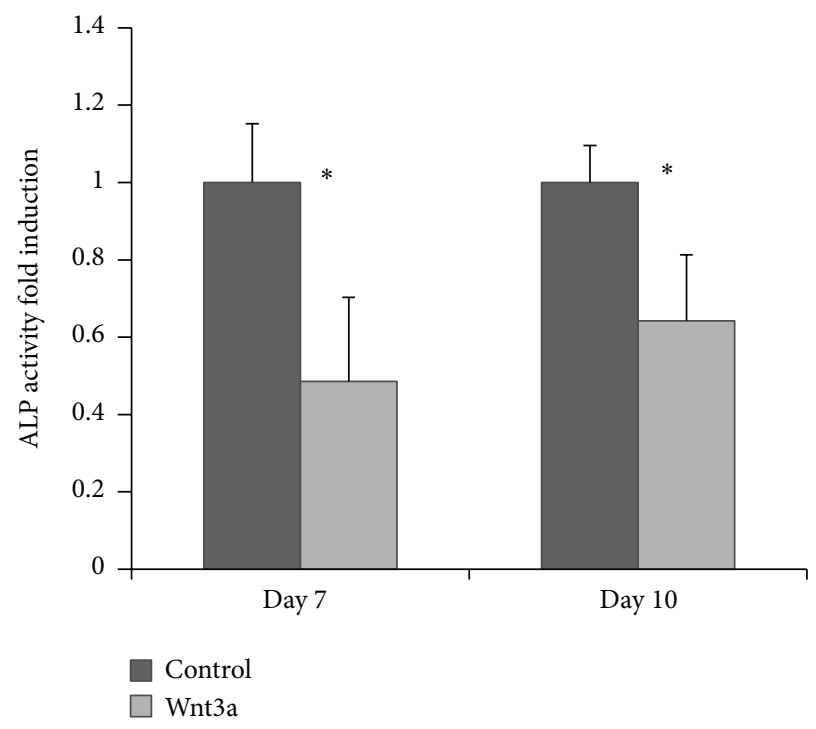

(c)

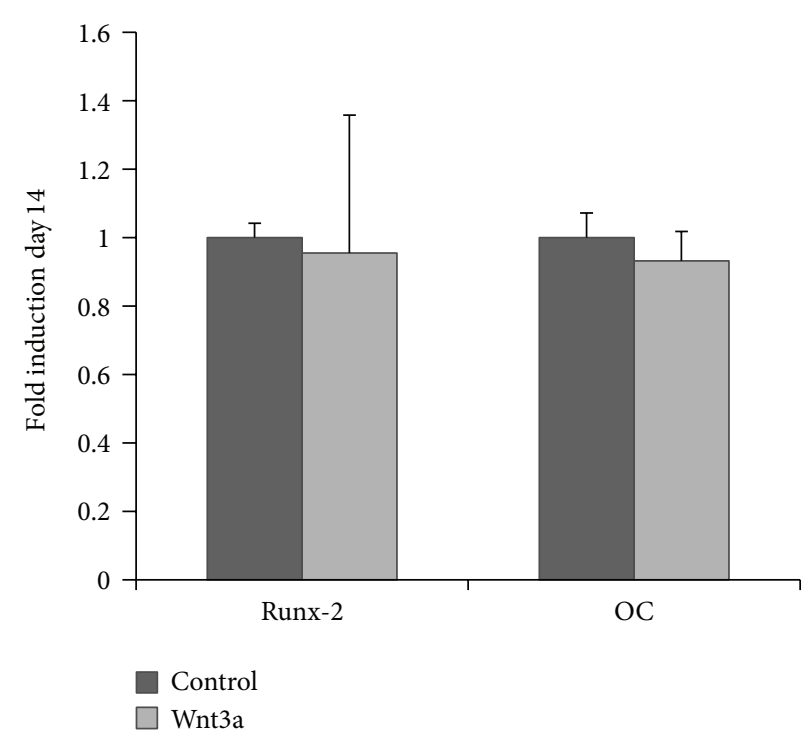

(b)

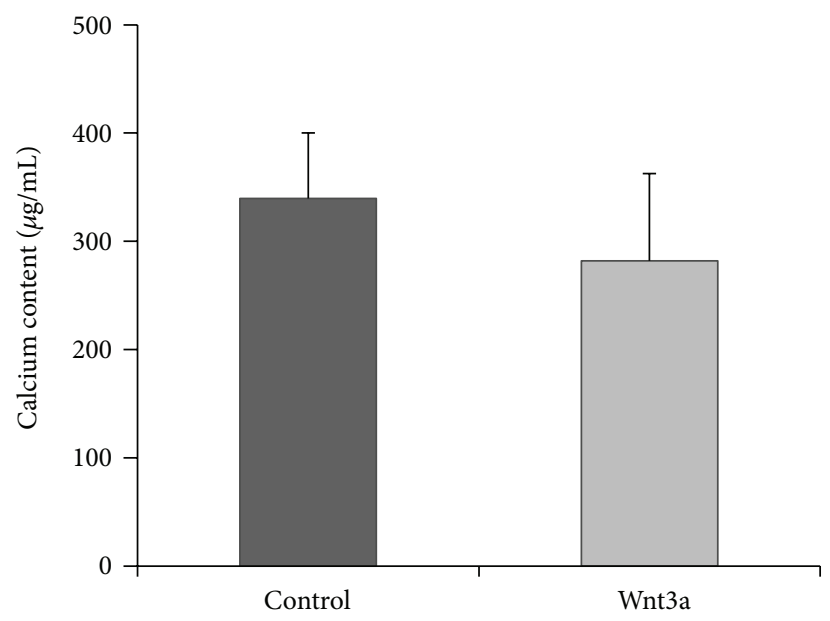

(d)

FIGURE 4: Representative results showed Wnt3a delayed osteogenic differentiation of hPDLCs. (a) ALP mRNA was downregulated by Wnt3a after 7 days of osteogenic induction. (b) The gene expression was equal for Runx-2 and OC between two groups. (c) ALP activity was downregulated by Wnt3a on days 7 and 10. (d) Wnt3a has no effect on the calcium content after 30 days of osteogenic induction. ${ }^{*} P<0.05$; error bars represent standard deviation.

ALP-activity were reduced significantly by Wnt3a during osteogenic differentiation. On the other hand, it has to be noted that for the mineralization, no effect of Wnt3a was observed after 30 days of culture, indicating Wnt3a may have a delayed effect on osteogenic differentiation of hPDLCs. The effect of Wnt signaling on the STRO- $1^{+}$cells in the efficiency of osteogenic differentiation was not evaluated here. Since cells selected by mesenchymal stem-cell marker STRO-1 are considered to be in an undifferentiated state, it can be speculated that enhanced activation of canonical Wnt signaling could also inhibit osteogenic differentiation of
STRO- $1^{+}$cells based on the previous study, where it has been demonstrated that enhanced activation of canonical Wnt signaling hindered osteogenic differentiation of undifferentiated MSCs. Interestingly, some in vivo studies reported that Wnt3a could enhance implant osseointegration [25] and promote a better healing of a calvarial defect in adult mice [24]. The enhancement of proliferation or self-renewal by Wnt3a may explain the acceleration of osteogenesis in vivo.

Recently Yamada et al. reported that XAV939, an inhibitor of canonical Wnt signaling, inhibited ALP activity of hPDLderived MSCs, indicating the promoting effect of canonical 


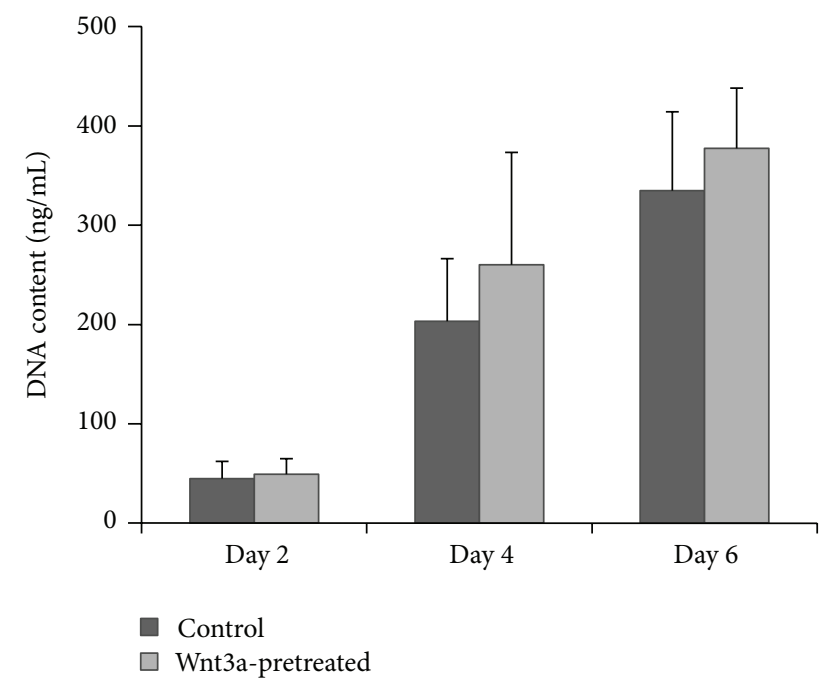

(a)

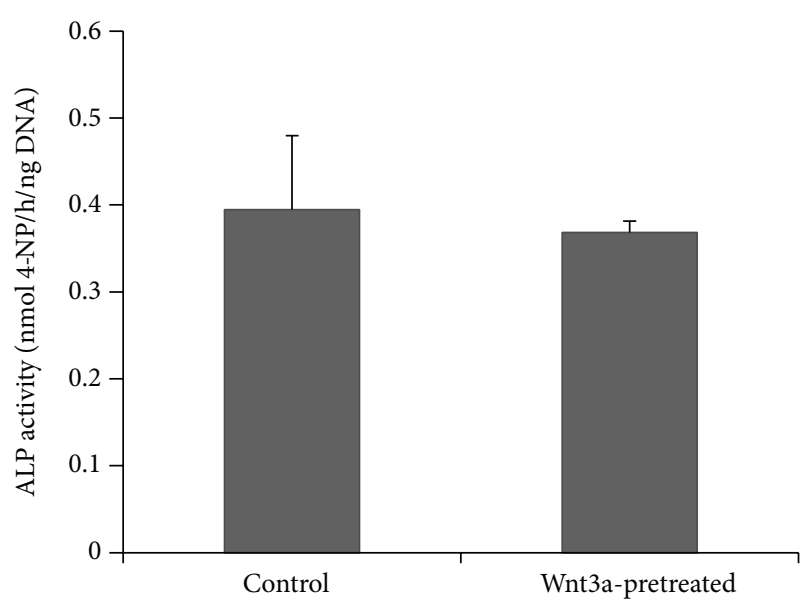

(c)

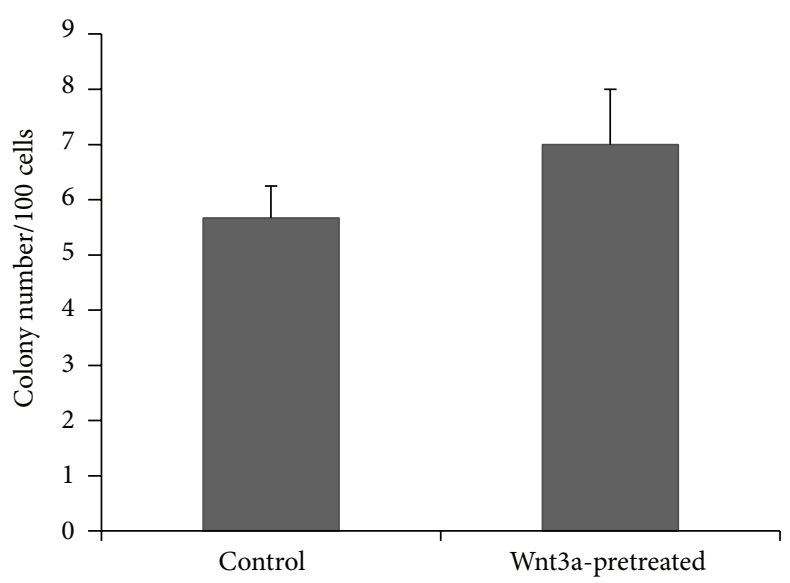

(b)

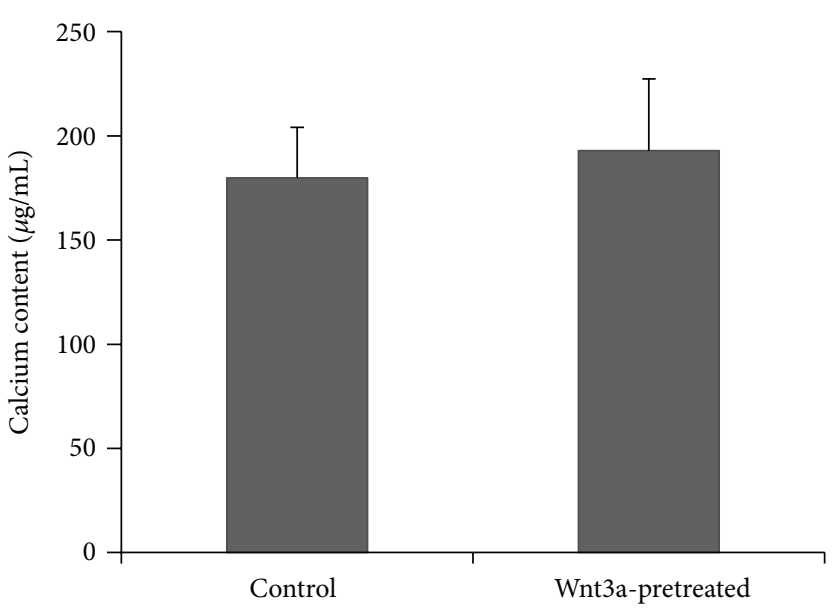

(d)

FIGURE 5: Representative results showed Wnt3a pretreatment did not affect functionality of hPDLCs after long-term expansion. (a) No

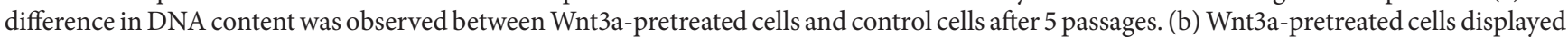
no difference compared to the control cells in CFU number. (c) No difference in ALP activity was observed between Wnt3a-pretreated cells and control cells. (d) No difference in calcium content was detected between Wnt3a-pretreated cells and control cells after 5 passages. Error bars represent standard deviation.

Wnt signaling pathway during the osteogenic differentiation of hPDLCs [29]. However, this mechanism is not consistent with the findings from this and also the other studies $[23,30]$. This discrepancy might be caused by the complexity of Wnt signaling. It has been reported that activation of canonical Wnt signaling may bring out opposite biological activity in the context of osteogenic differentiation depending on the threshold levels of its activation, the status of cell, and Wnt ligands concentration [24]. Therefore, the comprehensive understanding of the roles of both appropriate dose concentrations of Wnt3a treatment and endogenous activity of canonical Wnt signaling will be needed in further studies.

More hPDLCs with higher self-renewal ability can be obtained by using Wnt3a. To further confirm the functionality of Wnt3a expanded hPDLCs, we subcultured hPDLCs from passage 4 with or without Wnt3a up to passage 9 and compared the functionalities of Wnt3a-pretreated and control cells. Wnt3a promoted the rapid in vitro expansion of hPDLCs for at least 5 passages, as displayed by the shorter population doubling time, without interfering with their functionalities compared to untreated control cells. Wnt3a pretreatment resulted in much more hPDLCs with similar proliferation, CFU, and osteogenic capacity as the untreated control cells, which is of importance for the clinical use of hPDLCs because a large number of cells are required for cytotherapeutic purposes. In a previous study, Zeng and Nusse reported that Wnt3a could promote long-term expansion of mammary stem cells and maintain their self-renewal ability [22]. These data indicate that Wnt3a may be used as a useful culture supplement for the in vitro expansion of hPDLCs to benefit their clinical application. 


\section{Conclusions}

The proliferation, $\mathrm{CFU}$, and mineralization capacity of parental hPDLCs and expanded STRO-1-sorted cells were compared to find the appropriate PDL cell population for future clinical applications. The results provide evidence that STRO-1-sorted hPDLCs after expansion are not superior compared to their unsorted parental cells, suggesting the time-consuming cell selection and expansion procedure can be avoided in cell-based periodontal regeneration. In contrast, Wnt3a does have an effect on hPDLCs by promoting cell proliferation and self-renewal, as displayed by an increased DNA content, a shorter cell population doubling time, and higher expression of the self-renewal gene Oct4. In addition, Wnt3a promoted the efficient in vitro expansion of hPDLCs for at least 5 passages without affecting the self-renewal and osteogenic differentiation capacity, indicating its potential for rapid and efficient in vitro cell expansion for future clinical applications.

\section{Conflict of Interests}

The authors declare that there is no conflict of interests regarding the publication of this paper.

\section{Acknowledgments}

The authors thank China Scholarship Council (no. 2010622061) and Royal Netherlands Academy of Arts and Sciences (KNAW; project no. PSA 08-PSA-M-02).

\section{References}

[1] J. M. Albandar, "Epidemiology and risk factors of periodontal diseases," Dental Clinics of North America, vol. 49, no. 3, pp. 517532, 2005.

[2] V. Baelum and R. Lopez, "Periodontal disease epidemiologylearned and unlearned?” Periodontology 2000, vol. 62, no. 1, pp. 37-58, 2013.

[3] P. M. Bartold, C. A. G. McCulloch, A. S. Narayanan, and S. Pitaru, "Tissue engineering: a new paradigm for periodontal regeneration based on molecular and cell biology," Periodontology 2000, vol. 24, no. 1, pp. 253-269, 2000.

[4] F.-M. Chen and Y. Jin, "Periodontal tissue engineering and regeneration: current approaches and expanding opportunities," Tissue engineering B, vol. 16, no. 2, pp. 219-255, 2010.

[5] N. Yu, D. A. W. Oortgiesen, A. L. J. J. Bronckers, F. Yang, X. F. Walboomers, and J. A. Jansen, "Enhanced periodontal tissue regeneration by periodontal cell implantation," Journal of Clinical Periodontology, vol. 40, no. 7, pp. 698-706, 2013.

[6] Y. Tsumanuma, T. Iwata, K. Washio et al., "Comparison of different tissue-derived stem cell sheets for periodontal regeneration in a canine 1-wall defect model," Biomaterials, vol. 32, no. 25, pp. 5819-5825, 2011.

[7] S. J. Dangaria, Y. Ito, X. Luan, and T. G. H. Diekwisch, "Successful periodontal ligament regeneration by periodontal progenitor preseeding on natural tooth root surfaces," Stem Cells and Development, vol. 20, no. 10, pp. 1659-1668, 2011.
[8] B.-M. Seo, M. Miura, S. Gronthos et al., "Investigation of multipotent postnatal stem cells from human periodontal ligament," The Lancet, vol. 364, no. 9429, pp. 149-155, 2004.

[9] T. Nakahara, T. Nakamura, E. Kobayashi et al., "In situ tissue engineering of periodontal tissues by seeding with periodontal ligament-derived cells," Tissue Engineering, vol. 10, no. 3-4, pp. 537-544, 2004.

[10] A. Doğan, A. Özdemir, A. Kubar, and T. Oygür, "Healing of artificial fenestration defects by seeding of fibroblast-like cells derived from regenerated periodontal ligament in a dog: a preliminary study," Tissue Engineering, vol. 9, no. 6, pp. 11891196, 2003.

[11] W. Zhang, X. F. Walboomers, S. Shi, M. Fan, and J. A. Jansen, "Multilineage differentiation potential of stem cells derived from human dental pulp after cryopreservation," Tissue Engineering, vol. 12, no. 10, pp. 2813-2823, 2006.

[12] C.-Y. C. Huang, D. Pelaez, J. D. Bendala, F. Garcia-Godoy, and H. S. Cheung, "Plasticity of stem cells derived from adult periodontal ligament," Regenerative Medicine, vol. 4, no. 6, pp. 809-821, 2009.

[13] T. Itaya, H. Kagami, K. Okada et al., "Characteristic changes of periodontal ligament-derived cells during passage," Journal of Periodontal Research, vol. 44, no. 4, pp. 425-433, 2009.

[14] T. Iwata, M. Yamato, Z. Zhang et al., "Validation of human periodontal ligament-derived cells as a reliable source for cytotherapeutic use," Journal of Clinical Periodontology, vol. 37, no. 12, pp. 1088-1099, 2010.

[15] M. C. Kruyt, J. D. de Bruijn, H. Yuan et al., "Optimization of bone tissue engineering in goats: a peroperative seeding method using cryopreserved cells and localized bone formation in calcium phosphate scaffolds," Transplantation, vol. 77, no. 3, pp. 359-365, 2004.

[16] S. K. Both, A. J. C. van der Muijsenberg, C. A. van Blitterswijk, J. de Boer, and J. D. de Bruijn, "A rapid and efficient method for expansion of human mesenchymal stem cells," Tissue Engineering, vol. 13, no. 1, pp. 3-9, 2007.

[17] L. Sarkar and P. T. Sharpe, "Inhibition of Wnt signaling by exogenous Mfrzbl protein affects molar tooth size," Journal of Dental Research, vol. 79, no. 4, pp. 920-925, 2000.

[18] E. Järvinen, I. Salazar-Ciudad, W. Birchmeier, M. M. Taketo, J. Jernvall, and I. Thesleff, "Continuous tooth generation in mouse is induced by activated epithelial Wnt/ $\beta$-catenin signaling," Proceedings of the National Academy of Sciences of the United States of America, vol. 103, no. 49, pp. 18627-18632, 2006.

[19] S. M. Rooker, B. Liu, and J. A. Helms, "Role of Wnt signaling in the biology of the periodontium," Developmental Dynamics, vol. 239, no. 1, pp. 140-147, 2010.

[20] K. Willert, J. D. Brown, E. Danenberg et al., "Wnt proteins are lipid-modified and can act as stem cell growth factors," Nature, vol. 423, no. 6938, pp. 448-452, 2003.

[21] M. Y. S. Kalani, S. H. Cheshier, B. J. Cord et al., "Wnt-mediated self-renewal of neural stem/progenitor cells," Proceedings of the National Academy of Sciences of the United States of America, vol. 105, no. 44, pp. 16970-16975, 2008.

[22] Y. A. Zeng and R. Nusse, "Wnt proteins are self-renewal factors for mammary stem cells and promote their long-term expansion in culture," Cell Stem Cell, vol. 6, no. 6, pp. 568-577, 2010.

[23] G. M. Boland, G. Perkins, D. J. Hall, and R. S. Tuan, "Wnt 3a promotes proliferation and suppresses osteogenic differentiation of adult human mesenchymal stem cells," Journal of Cellular Biochemistry, vol. 93, no. 6, pp. 1210-1230, 2004. 
[24] N. Quarto, B. Behr, and M. T. Longaker, "Opposite spectrum of activity of canonical wnt signaling in the osteogenic context of undifferentiated and differentiated mesenchymal cells: implications for tissue engineering," Tissue Engineering A, vol. 16, no. 10, pp. 3185-3197, 2010.

[25] A. Popelut, S. M. Rooker, P. Leucht, M. Medio, J. B. Brunski, and J. A. Helms, "The acceleration of implant osseointegration by liposomal Wnt3a," Biomaterials, vol. 31, no. 35, pp. 9173-9181, 2010.

[26] Y.-H. Loh, Q. Wu, J.-L. Chew et al., “The Oct4 and Nanog transcription network regulates pluripotency in mouse embryonic stem cells," Nature Genetics, vol. 38, no. 4, pp. 431-440, 2006.

[27] O. Trubiani, S. F. Zalzal, R. Paganelli et al., "Expression profile of the embryonic markers nanog, OCT-4, SSEA-1, SSEA-4, and frizzled-9 receptor in human periodontal ligament mesenchymal stem cells," Journal of Cellular Physiology, vol. 225, no. 1, pp. 123-131, 2010.

[28] F. Yi, L. Pereira, J. A. Hoffman et al., "Opposing effects of Tcf3 and Tcfl control Wnt stimulation of embryonic stem cell selfrenewal," Nature Cell Biology, vol. 13, no. 7, pp. 762-770, 2011.

[29] A. Yamada, T. Iwata, M. Yamato, T. Okano, and Y. Izumi, "Diverse functions of secreted frizzled-related proteins in the osteoblastogenesis of human multipotent mesenchymal stromal cells," Biomaterials, vol. 34, no. 13, pp. 3270-3278, 2013.

[30] J. de Boer, R. Siddappa, C. Gaspar, A. van Apeldoorn, R. Fodde, and C. van Blitterswijk, "Wnt signaling inhibits osteogenic differentiation of human mesenchymal stem cells," Bone, vol. 34, no. 5, pp. 818-826, 2004. 


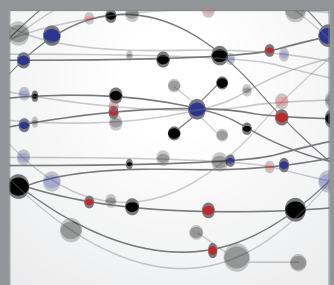

The Scientific World Journal
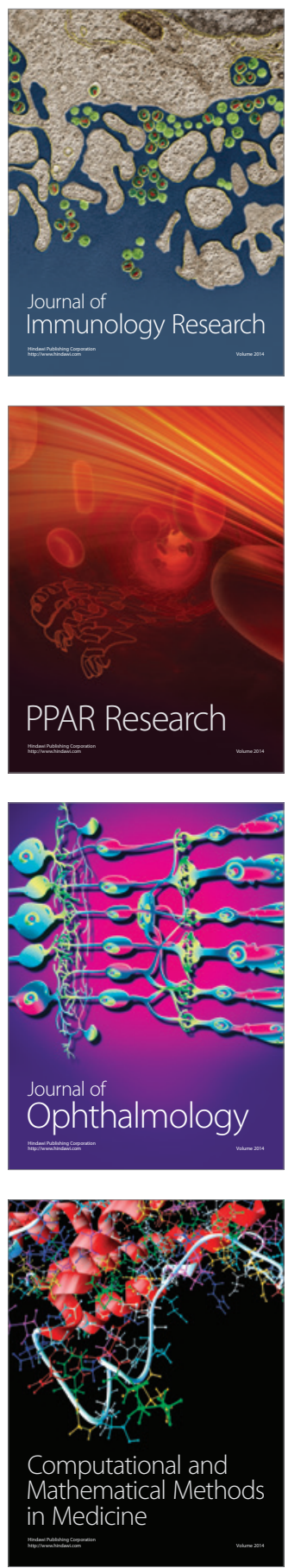

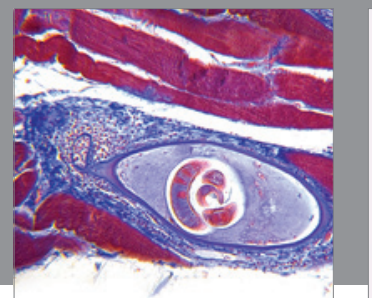

Gastroenterology

Research and Practice
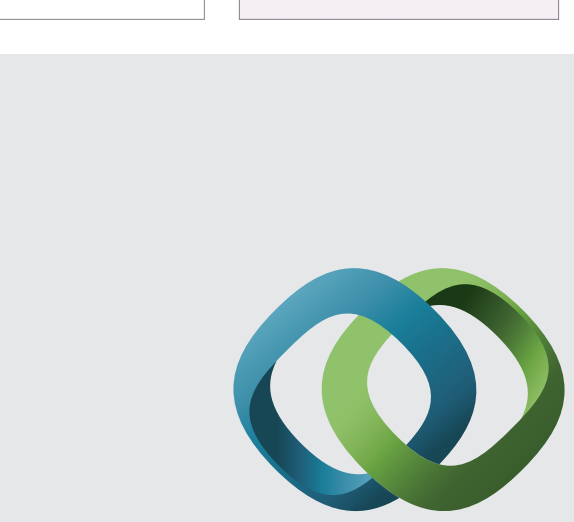

\section{Hindawi}

Submit your manuscripts at

http://www.hindawi.com
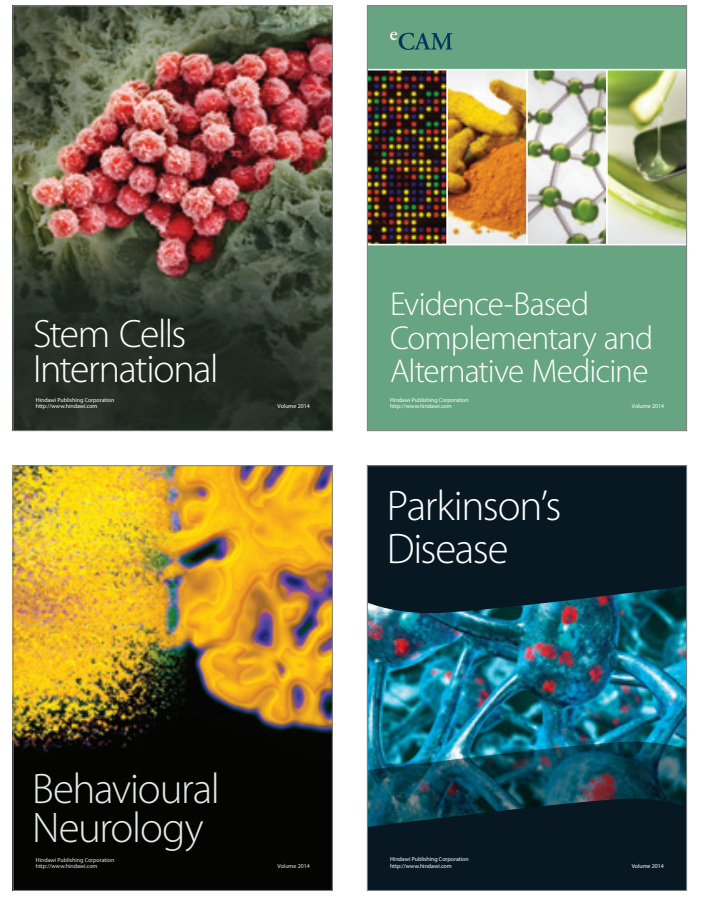
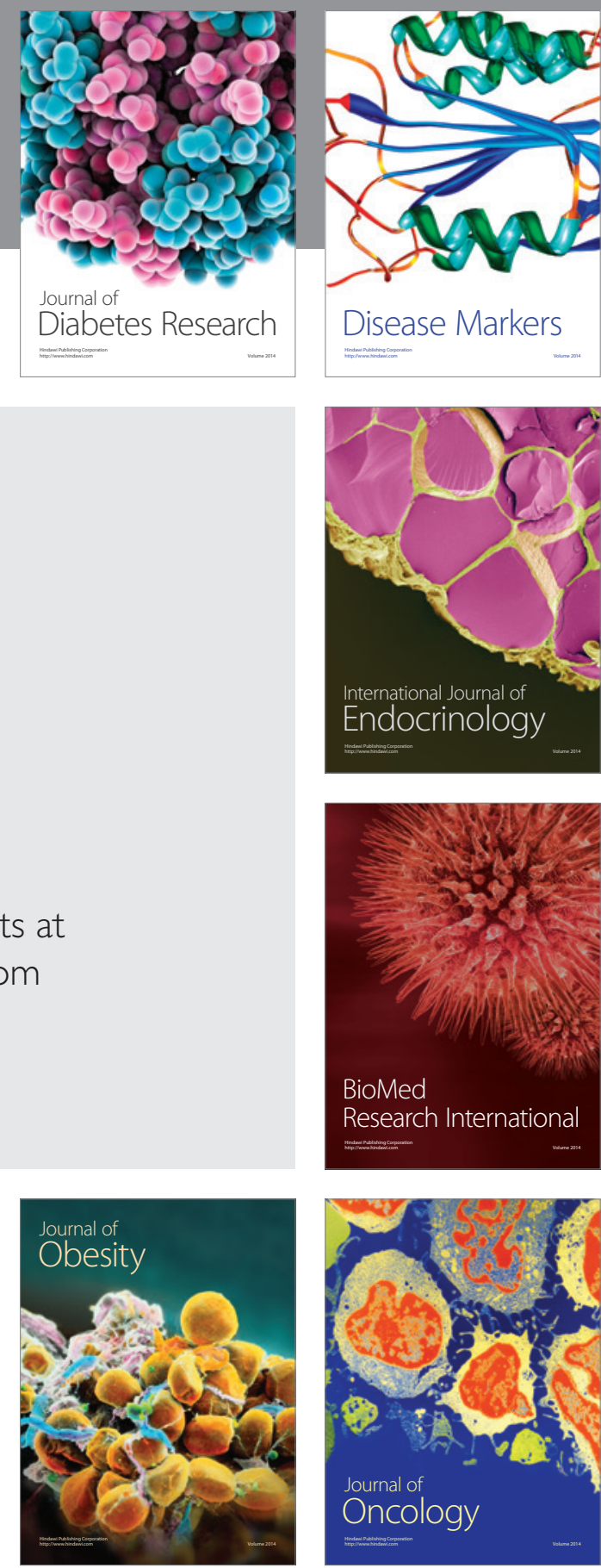

Disease Markers
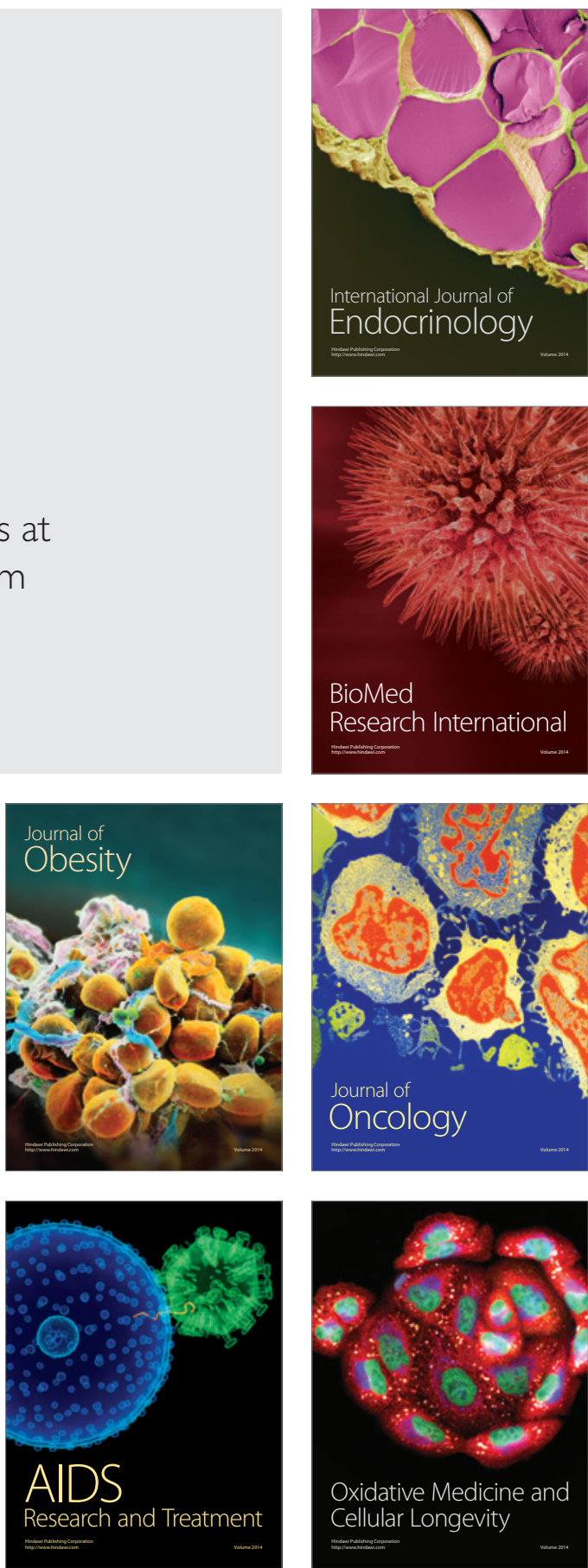